\title{
Retracted: Meta-Analysis of the Effectiveness and Safety of Intravenous Thrombolysis in Patients with Acute Cerebral Infarction
}

\author{
Computational and Mathematical Methods in Medicine \\ Received 24 November 2022; Accepted 24 November 2022; Published 25 December 2022 \\ Copyright (C) 2022 Computational and Mathematical Methods in Medicine. This is an open access article distributed under the \\ Creative Commons Attribution License, which permits unrestricted use, distribution, and reproduction in any medium, \\ provided the original work is properly cited.
}

Computational and Mathematical Methods in Medicine has retracted the article titled "Meta-Analysis of the Effectiveness and Safety of Intravenous Thrombolysis in Patients with Acute Cerebral Infarction" [1] due to concerns that the peer review process has been compromised.

Following an investigation conducted by the Hindawi Research Integrity team [2], significant concerns were identified with the peer reviewers assigned to this article; the investigation has concluded that the peer review process was compromised. We therefore can no longer trust the peer review process and the article is being retracted with the agreement of the Chief Editor.

The authors do not agree to the retraction.

\section{References}

[1] H. Wu, W. Gong, Y. Tang, W. Xu, Y. Zhou, and X. Liu, "MetaAnalysis of the Effectiveness and Safety of Intravenous Thrombolysis in Patients with Acute Cerebral Infarction," Computational and Mathematical Methods in Medicine, vol. 2021, Article ID 2833043, 11 pages, 2021.

[2] L. Ferguson, "Advancing Research Integrity Collaboratively and with Vigour,” 2022, https://www.hindawi.com/post/advancingresearch-integrity-collaboratively-and-vigour/. 


\title{
Meta-Analysis of the Effectiveness and Safety of Intravenous Thrombolysis in Patients with Acute Cerebral Infarction
}

\author{
Huili Wu, ${ }^{1}$ Weiping Gong $\mathbb{D}^{1}{ }^{1}$ Yanyan Tang, ${ }^{1}$ Wuhua $X{ }^{2},{ }^{2}$ Ying $\mathrm{Zhou}^{1}$ and Xianjun Liu ${ }^{1}$ \\ ${ }^{1}$ Department of Neurology, North Hospital of Yongzhou Central Hospital, Hunan 425000, China \\ ${ }^{2}$ Department of Rehabilitation Medicine, Guangzhou Red Cross Hospital, Guangdong 510000, China \\ Correspondence should be addressed to Weiping Gong; b20160905108@stu.ccsu.edu.cn
}

Received 3 November 2021; Revised 18 November 2021; Accepted 26 November 2021; Published 7 December 2021

Academic Editor: Osamah Ibrahim Khalaf

Copyright (C) 2021 Huili Wu et al. This is an open access article distributed under the Creative Commons Attribution License, which permits unrestricted use, distribution, and reproduction in any medium, provided the original work is properly cited.

\begin{abstract}
In this paper, a meta-analysis of the effectiveness and safety of intravenous thrombolysis in patients with acute cerebral infarction was carried out, the original literature inclusion criteria and retrieval strategies were developed, and the collection deadline was about new oral anticoagulants and other methods for the antithrombotic intravenous thrombolytic treatment of patients with acute cerebral infarction for the relevant literature on the safety and effectiveness comparison. First, the quality of the literature is evaluated according to whether the included studies are randomized controlled trials, whether there is randomized concealment, whether blinding is used, and whether they are withdrawn or lost to follow-up, and the RevMan 5.2 software is used for meta-analysis. At the same time, grey literature databases such as dissertations were experimentally searched, and all randomized controlled studies (RCT), nonrandomized controlled studies, case-controlled studies, cohort studies, case series reports, etc. of Wingspan in the treatment of intracranial atherosclerotic stenosis were collected. In the prevention of myocardial infarction in patients with acute cerebral infarction, the difference between the two was not statistically significant $(R R=0.82$, $95 \% \mathrm{CI}(0.57,1.17), P=0.27)$. Compared with other methods, it can significantly reduce the all-cause mortality of patients with nonvalvular venous thrombolysis, and the difference is statistically significant $(\mathrm{RR}=0.90,95 \% \mathrm{CI}(0.85,0.96), P=0.001)$. Experimental results show that in terms of safety, the new oral anticoagulant is better than other methods in reducing minor bleeding in patients with acute cerebral infarction, and the difference is statistically significant ( $\mathrm{RR}=0.87,95 \% \mathrm{CI}(0.76,099)$, $P=0.03$ ); the effect is better than other methods in reducing the incidence of serious bleeding events, and the difference is statistically significant $(\mathrm{RR}=0.79,95 \% \mathrm{CI}(0.74,0.85), P<0.00001)$.
\end{abstract}

\section{Introduction}

Intravenous thrombolysis is an independent risk factor for acute cerebral infarction. Acute cerebral infarction caused by intravenous thrombolysis has a high fatality and disability rate. At the same time, thrombus formed during intravenous thrombolysis can also cause pulmonary vein thrombolysis and myocardial infarction [1]. Thus, standardized anticoagulation medication is a critical component of the overall management of patients receiving intravenous thrombolysis. Other approaches are presently the most extensively used anticoagulant medications in clinical practise, but owing to considerable individual variations, it is required to regularly monitor coagulation-related indicators to modify the drug dosage during usage, which restricts their use to a certain degree [2-5]. At the moment, new oral anticoagulants such as dabigatran etexilate, rivaroxaban, apixaban, and edoxaban have been developed that exhibit steady effectiveness, good safety, and ease of administration. Foreign clinical trials have demonstrated that new oral anticoagulants are more effective than other methods at reducing severe bleeding in patients receiving nonvalvular intravenous thrombolysis, but they may increase the risk of gastrointestinal bleeding and do not prevent acute cerebral hemorrhage. Conclusions on the effectiveness of intravenous thrombolysis for infarction or thrombosis remain inconsistent [6-8].

Acute cerebral infarction repetitive transcranial magnetic stimulation, a painless and noninvasive brain stimulation tool, achieves its therapeutic effect by regulating the cortical excitability of the brain stimulation site and its rehabilitation 
treatment effect on the motor function of patients with acute cerebral infarction. It has been confirmed by a number of clinical studies [9]. Transcranial magnetic stimulation therapy has been shown to promote motor function recovery following acute cerebral infarction [10], and unlike transcranial magnetic stimulation therapy, repeated transcranial magnetic stimulation therapy is a continuous, adjustable, and safe clinical treatment measure. We focus on the effect of repetitive transcranial magnetic stimulation on motor function in patients with acute cerebral infarction in this systematic review and meta-analysis, using the FMA scale, grip strength, JTHF hand function test, Pap index/modified, and NIHSS scale as evaluation indexes [11]. The research and analysis revealed that the experimental group's FMA scores were much higher than those in the control group after repeated transcranial magnetic stimulation therapy, and the findings were statistically significant.

This paper's experimental quality evaluation results show that 4 articles are scored 6 points, 1 article is 5 points, and 3 articles are scored 4 points. The results of meta-analysis showed that in terms of effectiveness, new oral anticoagulants were superior to other methods in reducing the incidence of acute cerebral infarction in patients with acute cerebral infarction, and the difference was statistically significant $(\mathrm{RR}=0.83,95 \% \mathrm{CI}(0.71,0.97), P=0.02)$. The results of subgroup analysis showed that compared with other methods, new oral anticoagulants can significantly reduce the incidence of acute cerebral infarction in patients with acute cerebral infarction at high risk of acute cerebral infarction, and the difference is statistically significant $(R R=0.86,95 \% \mathrm{CI}$ (0.77, 0.96), $P=0.008)$ : compared with other methods, new oral anticoagulants can reduce the incidence of acute cerebral infarction in patients with acute cerebral infarction at a low risk of acute cerebral infarction, and there is a difference $(\mathrm{RR}=0.73,95 \% \mathrm{CI}(0.64,0.85), P<0.001)$.

\section{Related Work}

The Fugl-Meyer Rating Scale (FMA) change before and after therapy is the associated assessment indicator. The change in grip strength before and after therapy, the change in the Jebsen Taylor Hand Function Test Scale (JTHF) before and after treatment, and the change in the Pap index before and after treatment are the secondary assessment indices. It examines changes in the modified Pap index and the National Institutes of Health Acute Cerebral Infarction Scale (NIHSS) before and during therapy. Clinical investigations using both high-frequency transcranial magnetic stimulation $(>1 \mathrm{~Hz})$ and low-frequency transcranial magnetic stimulation $(1 \mathrm{~Hz})$ as intervention strategies may be included to reach a more complete outcome.

Intravenous thrombolytic expansion has been shown to be a successful and safe therapeutic method for ICAD in many retrospective case studies [12]. PTA, on the other hand, has several technical limitations, including elastic retraction of the arteries and a high rate of restenosis. Bypass surgery is less successful than medication therapy for middle cerebral artery stenosis. While some trials have shown that coronary venous thrombolytic expansion stents are safe and effective for PTAS, others have demonstrated a significant risk of perioperative complications (23\%) and death (5\%) [13]. Since then, several facilities across the globe have done safety studies using Wingspan stents. By comparing the Wingspan stent's decreased perioperative complication rate to the increased risk of recurrence of acute cerebral infarction in the WASID trial, the stent's safety has been shown [14]. However, the results of the SAMMPRIS test suggest that the therapeutic drug group for intracranial atherosclerotic stenosis is better than the Wingspan stent group. Subsequently, transcranial magnetic stimulation has gradually been used in many fields such as clinical diagnosis, curative effect evaluation, and treatment. Cranial magnetic stimulation therapy is a new type of physical therapy that acts on the brain through electromagnetic effects, by changing changes in cerebral blood flow, the activity of partial enzymes in neuronal cells, the interaction between cortex and subcortical structures, and cellular genes, thereby affecting neurotransmitter and synaptic plasticity, and further promoting nerve repair [15]. The treatment machine usually consists of a high-current pulse generator that can generate thousands of amperes of discharge current through a stimulation coil to generate a short magnetic field pulse, the strength of which can reach several tesla.

Wang et al.'s [16] study is a prospective nonrandomized controlled trial, and it includes a control group in accordance with SAMMPRIS, but its randomization procedure, allocation concealment, and blinding are not stated; Katsanos et al.'s [17] study is a randomized control trial, but the sample size is small, and the random technique is the random number table; nonetheless, the blinding method, allocation concealment, selection report, loss to follow-up, and other biases are not at risk. The basic characteristics and information of all included studies are described in the article. Li et al. [18] will extract the outcome indicators of all included studies and conduct a single-group rate metaanalysis. Karaszewski et al. [19] analyzed the data, of which $19.88 \%$ had intracranial carotid artery stenosis, $18.73 \%$ had extracranial vascular stenosis, $33.33 \%$ had both intracranial and extracranial stenosis, and 749 had intracranial artery stenosis. There were a total of 2002 stenosis sites in the patients, 170 cases (16.33\%) of single stenosis, and 579 cases (55.62\%) of multiple stenosis; in the single stenosis group, intracranial stenosis accounted for $54.12 \%$; in the multiple stenosis group, intracranial stenosis accounted for $48.87 \%$ $(P=0.127)$; Wang et al. [20] also found that severe stenosis (70-99\%) and occlusive lesions were more common in intracranial stenosis $(P=0.018)$.

\section{Construction of a Metamodel Based on the Effectiveness and Safety of Intravenous Thrombolysis in Patients with Acute Cerebral Infarction}

3.1. Pathological Characteristics of Acute Cerebral Infarction. Acute cerebral infarction is composed of the ischemic necrosis area in the center of the infarct focus and the ischemic edema area around the focus or called the ischemic 
penumbra area. The ischemic penumbra is defined as a reversible brain tissue injury in which the electrophysiological activity is stopped while the energy maintains the function of the ion pump and the cell structure is intact. Figure 1 shows the pathological structure of acute cerebral infarction.

Although the nerve cells in the ischemic necrosis zone of the infarct focus are irreversibly damaged, there is still a significant amount of collateral circulation in the ischemic edema zone or ischemic penumbra zone, which will continue to receive a significant amount of blood, despite the fact that these brain tissues have been irreversibly damaged. While there will be some degree of damage and related neurological abnormalities, these injured nerve cells may still live for a limited length of time and their function is still recoverable. If blood flow to these locations is restored rapidly enough within the effective time window, the metabolic problem of brain tissue may be corrected, nerve cells can continue to live, and the deficient nerve function can be restored.

In light of these pathophysiological factors, prompt recanalization of occluded blood arteries has emerged as the most safe and successful approach of treating acute cerebral infarction. When an occluded cerebrovascular system is reopened, blood circulation to the corresponding brain tissue is restored, allowing threatened nerve cells to be salvaged in time. This procedure may be used to treat patients with acute cerebral infarction and improve their prognosis. The pathological aspects of acute cerebral infarction are summarised in Table 1.

The current treatment options for acute cerebral infarction include thrombolysis, defibrosis, improvement of brain microcirculation, antiplatelet aggregation, promotion of collateral circulation regeneration, and nourishment of nerves, and thrombolytic treatment is especially effective in the effective time window. Early thrombolytic therapy is the safest and most effective method for the treatment of acute ischemic cerebral infarction in the world.

3.2. Local Intervention of Intravenous Thrombolysis. In the local intervention process of intravenous thrombolysis, firstly, we select the intracranial segment of internal carotid artery, middle cerebral artery M1 segment, anterior cerebral artery A1 segment, vertebral artery intracranial segment, basilar artery, and posterior cerebral artery P1 segment from the DSA angiography report, with stenosis for $70-99 \%$ of cases; the backup images were retrieved; and all images were reviewed independently by 2 neuroimaging doctors with more than 10 years of clinical experience. The degree of stenosis measures the point where the stenosis is the most severe compared with the normal blood vessel at the distal end of the stenosis (normal distal vessel diameter-residual lumen diameter/normal distal lumen diameter), or when the distal end of the stenosis is expanded or bifurcated, the stenosis is measured for the diameter of the proximal normal pipe diameter. Diffuse intracranial artery disease refers to the standard of Kumar and Alexandrov [21], which is multiple intracranial artery stenosis and multiple stenosis in one artery. We exclude cases that do not meet the criteria and mark the stenosis. Figure 2 is a chi-square test analysis of local intervention for intravenous thrombolysis.

In this study, Stata 14.0 was used to conduct traditional meta-analysis of direct comparison between pairs. The network meta-analysis based on Bayesian method uses GeMTC software for statistical analysis. The odds ratio (OR) and its 95\% CI are used as the effect size indicator. The chi-square test was used to analyze the heterogeneity between the results, and the $I^{2}$ quantitatively judged the magnitude of the heterogeneity. When the $I^{2}$ value is less than $25 \%$, it indicates that there is no heterogeneity; when the $I^{2}$ value is within $25 \%-50 \%$, it indicates that there is slight heterogeneity; when the $I^{2}$ value is within $50 \%-75 \%$, it indicates that there is large heterogeneity; when the $I^{2}$ value is greater than $75 \%$, it indicates a large heterogeneity. If the $I^{2}$ value is less than $50 \%$, a fixed-effects model is used for meta-analysis; if the $I^{2}$ value is greater than $50 \%$, after excluding obvious clinical heterogeneity, a random-effects model is used for meta-analysis. Only studies with the most complete data or the most recent years are included for studies with repeated publications or overlapping data. For documents that meet the inclusion criteria, the extracted data include title, author, research name, publication time, country or region, number of research subjects, race, gender, average age, diagnosis, onset time, follow-up time, various studies' medium antiplatelet regimen and dose, and the number of cases with outcome indicators.

3.3. Effectiveness and Safety Screening. Intravenous thrombolysis epidemiology bias says that research with statistical significance (results that are favourable) is easier to publish than research with no statistical significance (research with negative results). As a result, publication bias will be introduced into the research that is eventually included in the meta-analysis, resulting in a final conclusion that does not accurately reflect the relationship between treatment effect size and relevant risk factors and ultimately results in research conclusions that cannot accurately guide clinical practise. If the number of cases included in linked studies is considerable (more than ten), a funnel chart may be used to determine whether the related studies included in the analysis exhibit publication bias. A funnel chart is a scatter plot constructed using the logarithm of the effect size and the reciprocal of the effect size's standard error. It is possible to evaluate whether the included studies are biased by observing whether the funnel chart is symmetrical. If the asymmetric funnel chart is more obvious, the more the included studies are biased. Figure 3 is an analysis of the apparent bias in the effectiveness of intravenous thrombolysis.

Stenosis and obstructive lesions of the aorta and the arterial blood supply of the limbs, confirmed by color Doppler ultrasound or DSA, and atherosclerotic lesions are the cause. The frequency and/or rhythm of heart beats are abnormal due to the origin of heart activity and/or conduction disorders. Noninvasive and invasive examinations such as routine electrocardiogram, echocardiogram, electrocardiogram exercise stress test, radionuclide imaging, and cardioangiography confirm or exclude organic 


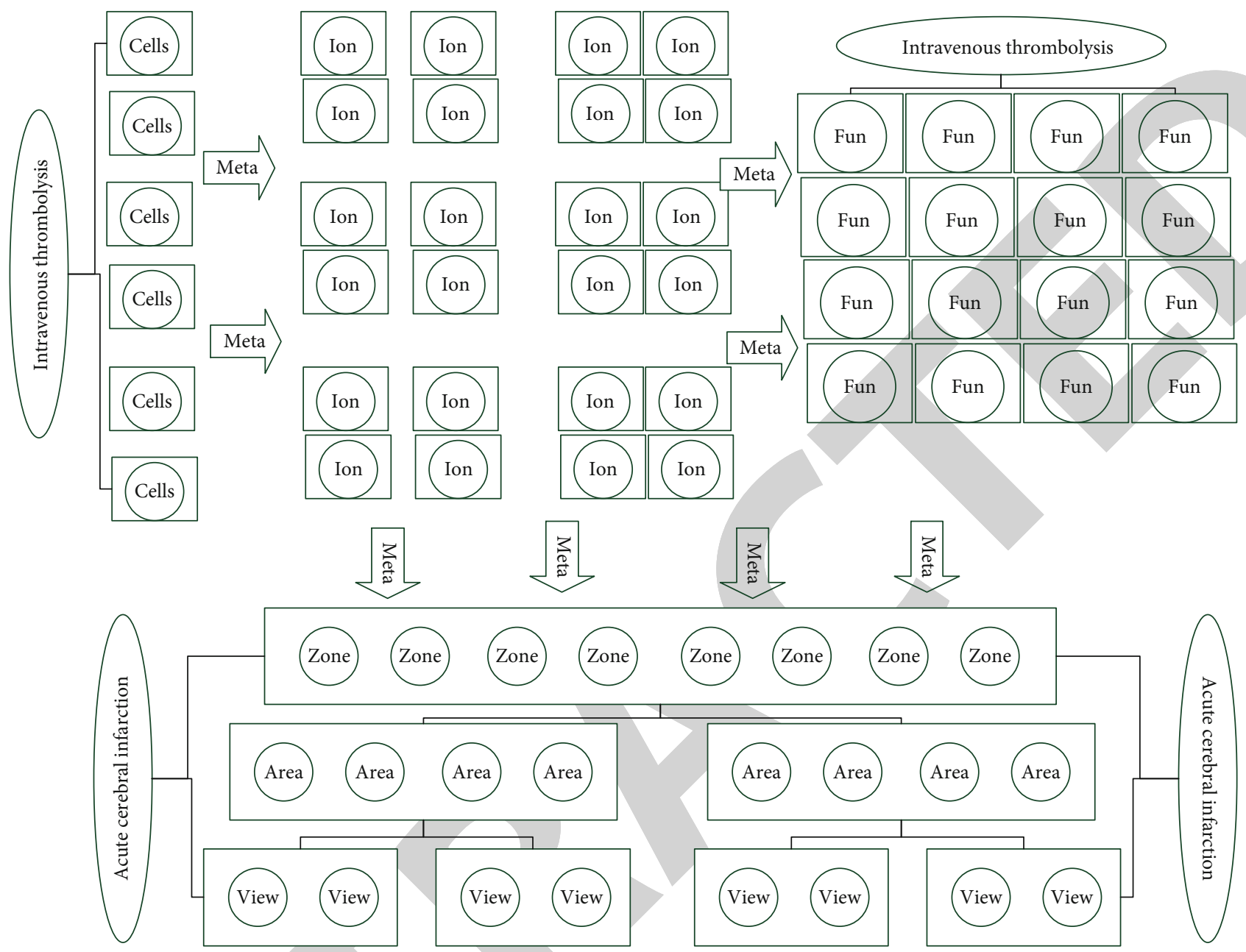

FIGURE 1: Pathological structure of acute cerebral infarction.

TABLE 1: Description of pathological features of acute cerebral infarction.

\begin{tabular}{|c|c|c|}
\hline Index number & Second cell indicator & Third cell indicator \\
\hline \multirow{3}{*}{ Pathological capability } & Return on infarction & 0.21 \\
\hline & Infarction turnover rate & 0.33 \\
\hline & Operating infarction growth rate & 0.15 \\
\hline & Infarction chain distribution accuracy & 0.09 \\
\hline Customer service ability & On-time rate of infarction chain distribution & 0.31 \\
\hline & Logistics service integration & 0.26 \\
\hline \multirow{2}{*}{3} & Information treatment & 0.28 \\
\hline & Information treatment degree & 0.54 \\
\hline
\end{tabular}

heart disease. The pathophysiological mechanism of progressive acute cerebral infarction includes hemodynamic mechanism, brain mechanism, and system mechanism. Batroxobin can decrease plasma viscosity, improve blood rheology, increase blood flow, and act as a scavenger of free radicals and antioxidants. Therefore, its therapeutic targets for progressive acute cerebral infarction may be to dissolve new small thrombi or emboli and improve microcirculation, while also delaying or preventing intravenous thrombolysis.
The thrombosis extends to the proximal end, that is, to the upper level of blood vessels, thereby helping to establish collateral circulation and prevent insufficient tissue perfusion in a larger area.

3.4. Metaquality Evaluation Standard. All data of metaquality evaluation were analyzed by SPSS 16.0 statistical software. Measurement data are expressed as $x \pm s$ or $M$ (Q25, Q75) and are compared using $t$-test or Mann-Whitney 


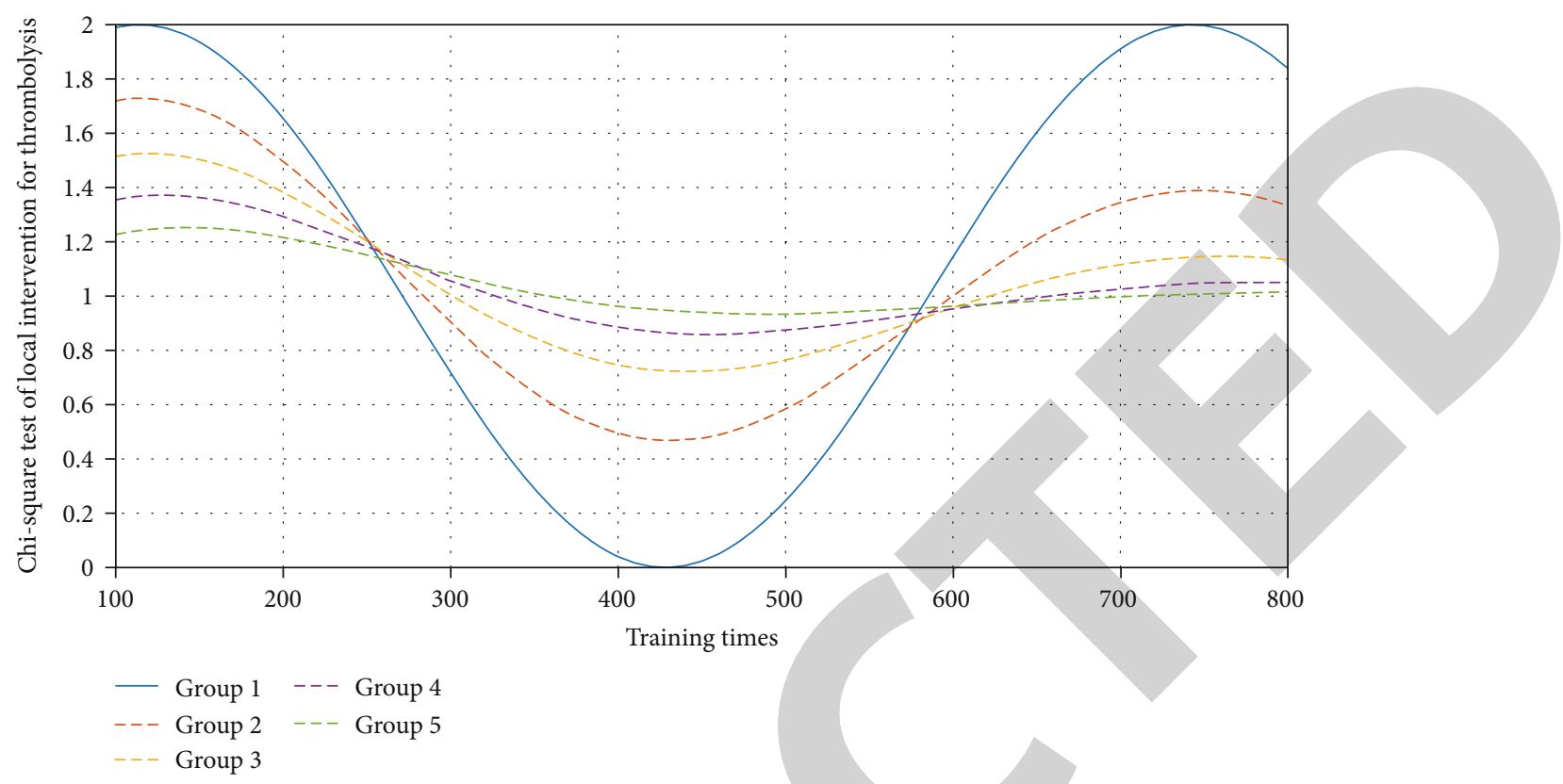

FIGURE 2: Chi-square test analysis of local intervention for intravenous thrombolysis.

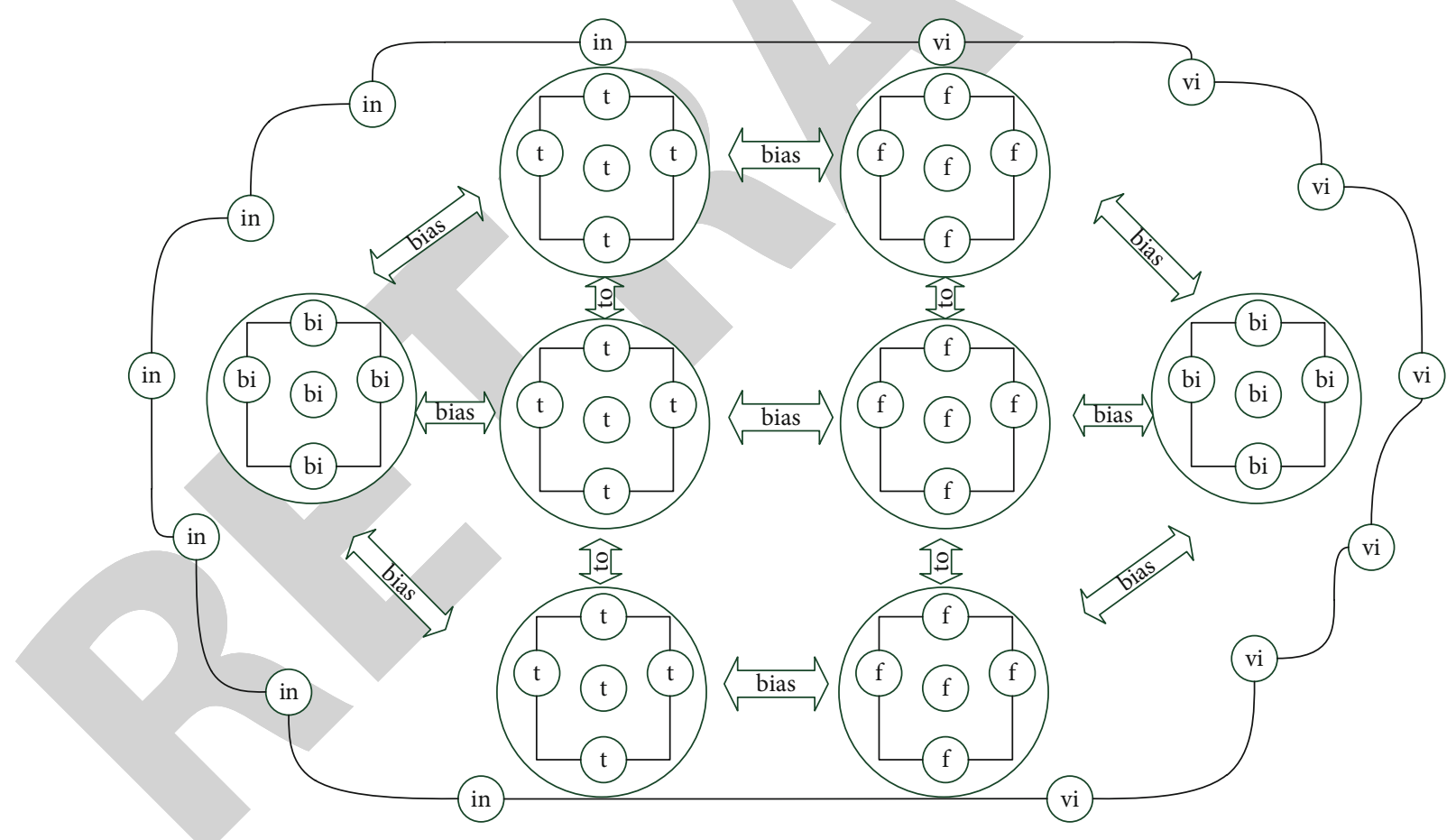

FIgURE 3: The analysis of the apparent bias in the effectiveness of intravenous thrombolysis.

$U$ test; count data are expressed as frequency or percentage, using the chi-square test or Fisher's exact probability method and using Kaplan-Meier method to estimate it. The incidence of end-point events at each time point and the median survival time were described. Log-rank test was used to compare the incidence between groups. Cox regression analysis was used to analyze the impact of each influencing factor on survival time. After browsing the title and abstract, 273 irrelevant documents were excluded, 246 duplicate documents were excluded, and 412 were left, including 2 in Chinese and English and 410 in Chinese. After further screening, 165 articles of non-RCT and combination medication were excluded, 132 articles of other uncertain doses of Batroxobin and other types of acute cerebral infarction were 


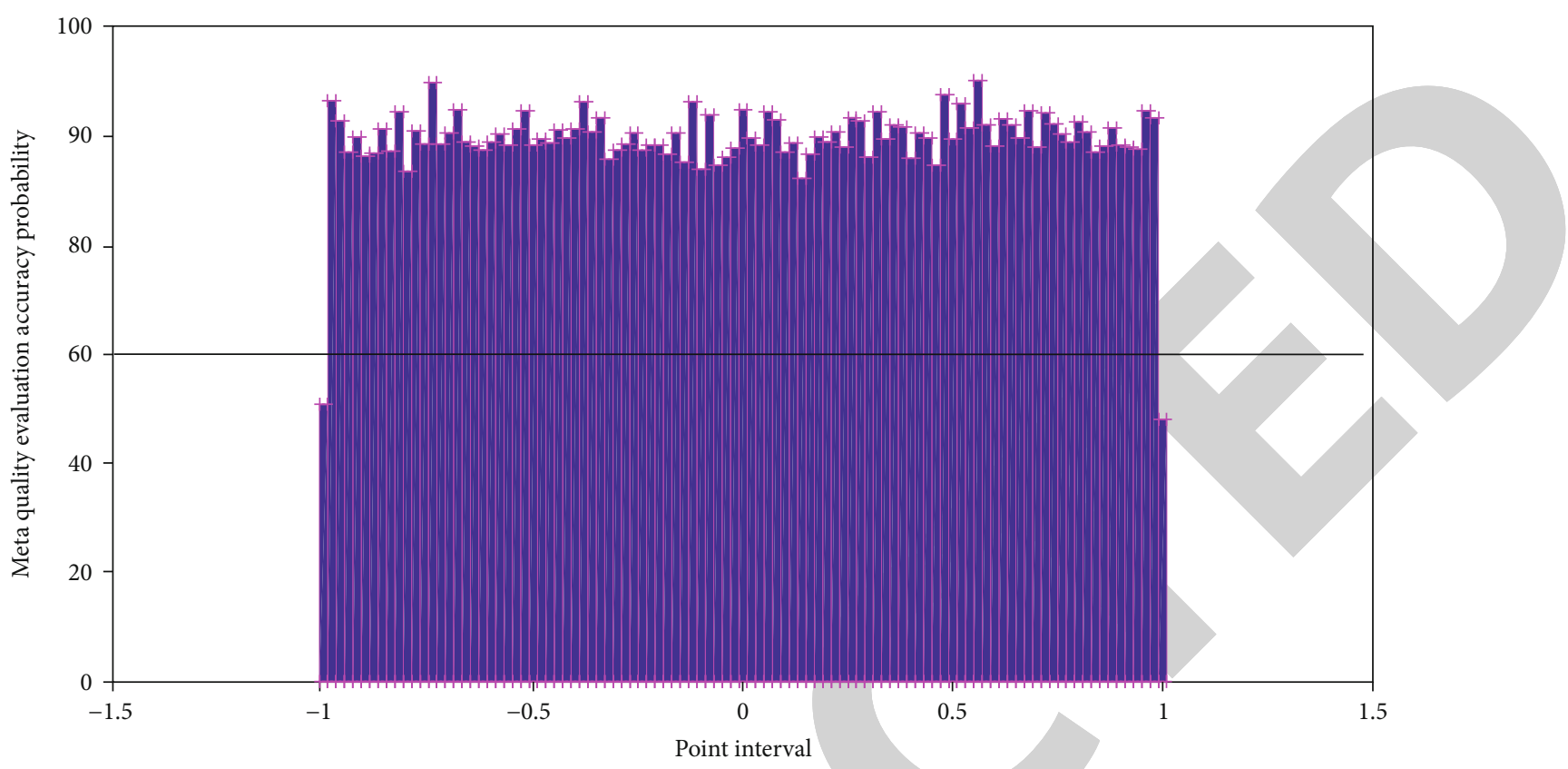

FIGURE 4: The accurate probability distribution of metaquality evaluation.

excluded, and 115 articles were left, including 1 article in English and 114 articles in Chinese. Figure 4 is the accurate probability distribution of metaquality evaluation.

During the average follow-up period of 36.6 months, the total incidence of the primary endpoint of the drug group was $39 / 172(22.67 \%)$, the 30 -day incidence was $14 / 172$ $(8.14 \%)$, and the 1 -year incidence was $28 / 172(16.28 \%)$. Kaplan-Meier analysis showed that, among the primary endpoint events, the median survival time (95\% confidence interval) of the surgery group and the drug group was $54.15(46.538,61.770)$ and $62.015(56.745,67.284)$, respectively. The log-rank test was used to compare the chisquare value which is $0.073, P=0.787$. There was no statistical difference between the two groups. The bias of the included studies was evaluated using the Cochrane Collaboration tool. Each study included in the analysis was evaluated in terms of selection bias, implementation bias, loss to followup bias, reporting bias, and other biases. Among the 26 included studies, 24 studies specifically described the generation of random sequences; 22 studies concealed the allocation plan; 2 studies did not blind the participants, implementers, and outcome measurers. The data are complete, and only 1 study may have reporting bias.

\section{Application and Analysis of Metamodel Based on the Effectiveness and Safety of Intravenous Thrombolysis in Patients with Acute Cerebral Infarction}

4.1. Metadata Preprocessing for Intravenous Thrombolysis. The meta-analysis in this article uses a single-group rate analysis method, adding weights to the analysis factors and obtaining indicators. Compared with the final data stent group results of SAMMPRIS, the surgical success rate of this study is $96.75 \%$ vs. $92.9 \%$, and the 30 -day end-point event rate was $8.75 \%$ vs. $14.7 \%$, the 1 -year end-point event rate was $13 \%$ vs. $19.7 \%$, and the mortality rate was $2.98 \%$ vs. $4 \%$. It indicated that the overall safety of surgery was better than that of the SAMMPRIS experiment. At the same time, the SAMMPRIS study showed that after 30 days, the primary endpoint event occurred in 21/210 (10\%) patients in the stent group and 19/191 (10\%) patients in the stent group, and the incidence of primary endpoint events was the same in the two groups. The Stata software will analyze the inconsistency in the closed loop, calculate the inconsistency factor (IF), and perform a $Z$ test. If $P>0.05$, there is no inconsistency. The studies included in the four outcome indicators were tested for inconsistency. After testing, no significant inconsistency was found $(P>0.05)$, so the consistency model was selected. Figure 5 shows the consistency of the metadata for intravenous thrombolysis.

After medical record retrieval and DSA report query, 307 patients who met the degree of vascular stenosis were listed. After the inclusion and exclusion criteria were compared, a total of 261 cases met the inclusion criteria, of which 41 patients could not be contacted and lost follow-up. In the end, 220 cases were selected, including 48 cases in the interventional group and 172 cases in the drug group. Three instances of intravenous thrombolytic expansion, 24 cases of intravenous thrombolytic expansion stents, and 21 cases of self-expanding stents were reported in the surgical group. There were no statistically significant variations in the degree of stenosis of the relevant blood arteries, vascular risk factors, or duration of follow-up; however, there were statistically significant disparities in stroke history. The metaanalysis of the RCT study found that when the Wingspan stent treatment group was compared to the drug group, 


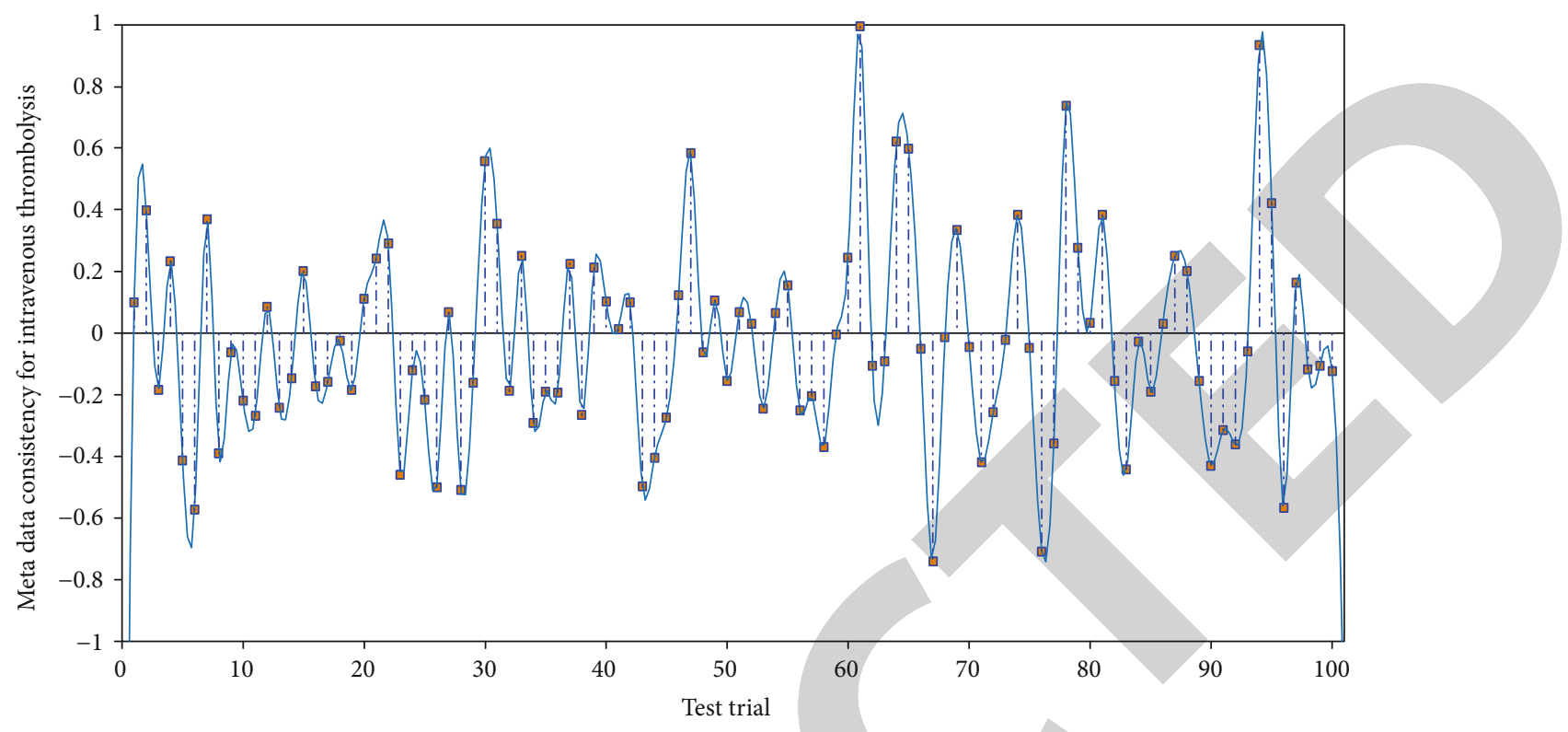

FIgURE 5: Metadata consistency of intravenous thrombolysis.

the drug group had a lower 30-day endpoint event rate than the stent group, with a statistically significant difference between the two groups; there was no difference in the 1year endpoint event rate between the two groups. The results of the meta-analysis of the single-group rate of all studies on the effectiveness and safety of Wingspan stent treatment are as follows: the surgical success rate was $96.75 \%$, the 30 -day endpoint event rate was $8.75 \%$, the 1 -year endpoint event rate was $13 \%$, and the overall mortality rate of in-stent restenosis was $2.98 \%$, the rate of in-stent restenosis was $21.76 \%$, the rate of symptomatic restenosis in the total number of observations was $6.5 \%$, and the rate of symptomatic restenosis in the number of restenosis was $26.06 \%$.

4.2. Simulation of the Effectiveness and Safety of Intravenous Thrombolysis. The simulation experiment on the effectiveness and safety of intravenous thrombolysis uses the RevMan5.2 software provided by the Cochrane Collaboration and the open-source and free $\mathrm{R}$ software for meta-analysis. The enumeration data uses relative risk or ratio $(R R)$ analysis and statistics, and the measurement data uses mean difference (MD) analysis and statistics. The significance level is designed as $\alpha=0.05$, and both have $95 \%$ confidence interval (CI). $I^{2}$ is used to quantitatively analyze the heterogeneity. If $I^{2}>50 \%$, it is considered that there is heterogeneity. Under the premise that there is no clinical heterogeneity between trials, when there is statistical heterogeneity, the randomeffects model is adopted, otherwise, the fixed-effects model is adopted. If there is clinical heterogeneity, the combined analysis is abandoned. We use funnel plots and egger funnel plots to test potential publication bias. At the same time, to ensure the reliability of the meta-analysis results, different statistical models can be used or low-quality literature can be removed for sensitivity analysis. Figure 6 is the sensitivity analysis of the effectiveness of intravenous thrombolysis.
The heterogeneity test is used to test the methodology and statistics between the included studies. Only the studies with low heterogeneity can be combined for statistics. In this study, the $I^{2}$ test was used to test the heterogeneity of the included literature. When $I^{2}<25 \%$, it indicates that the heterogeneity between the studies is relatively small; when $I^{2}>50 \%$, it indicates that there is a high degree of heterogeneity in the research results, $25 \%<I^{2}<50 \%$ shell. It is a moderate degree of heterogeneity. If the heterogeneity between studies is relatively high, subgroup analysis or sensitivity analysis can be used for the trial. It is necessary to exclude studies that may have abnormal results and then perform a meta-analysis to observe whether the results obtained have changed, thereby increasing the reliability and stability of the results. If the results are not significantly different after excluding the literature, it indicates that the results of the meta-analysis are reliable. Figure 7 shows the heterogeneity test of intravenous thrombolysis data.

A total of 732 patients meeting the criteria were included in this study, including 440 males and 292 females. The gender distribution of each subtype was statistically different $(P=0.002)$. Except for $C E$ subtypes, which were more common in women, all other subtypes were men are more common. The minimum age of onset was 31 years old, the maximum age of onset was 93 years old, and the average age of onset was $69.02 \pm 11.55$ years. There was a statistically significant difference in the age distribution between subtypes $(P=0.001)$. The age of CE subtype was larger than that of other subtypes. The plaque in the direction of the perforating vessels was regarded as a risk factor for surgery. The surgery group and the drug group accounted for $3 / 17(17.6 \%)$ and $18 / 35$ (51.4\%), respectively, $P=0.034$; the plaque enhancement group accounted for $10 /$ $17(58.8 \%)$ and $20 / 35$ (57.1\%), $P=0.908$; T1 hypersignal two groups accounted for $2 / 17(11.8 \%)$ and $9 / 35(25.7 \%)$, 


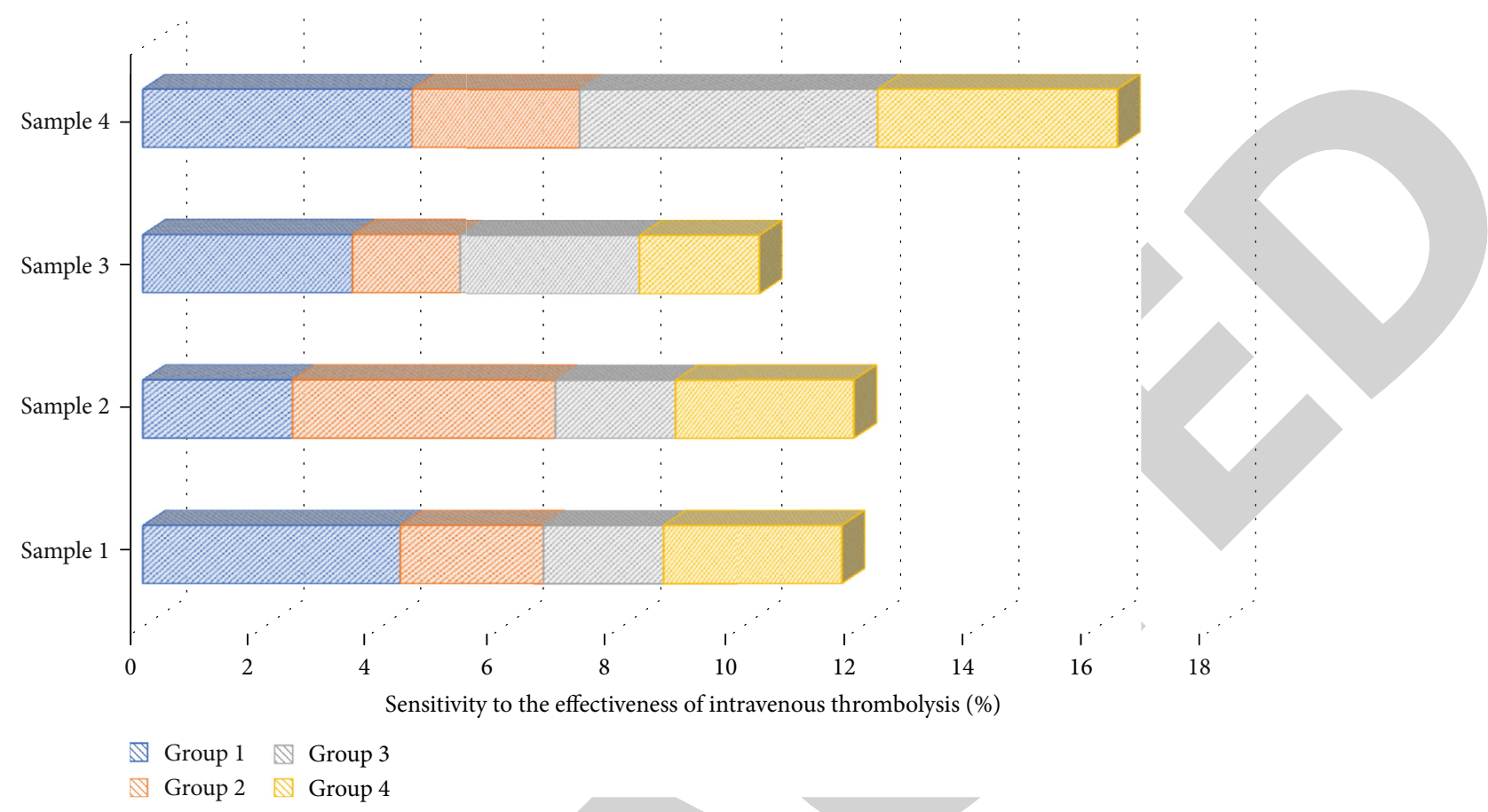

FIgURE 6: Sensitivity analysis of the effectiveness of intravenous thrombolysis.

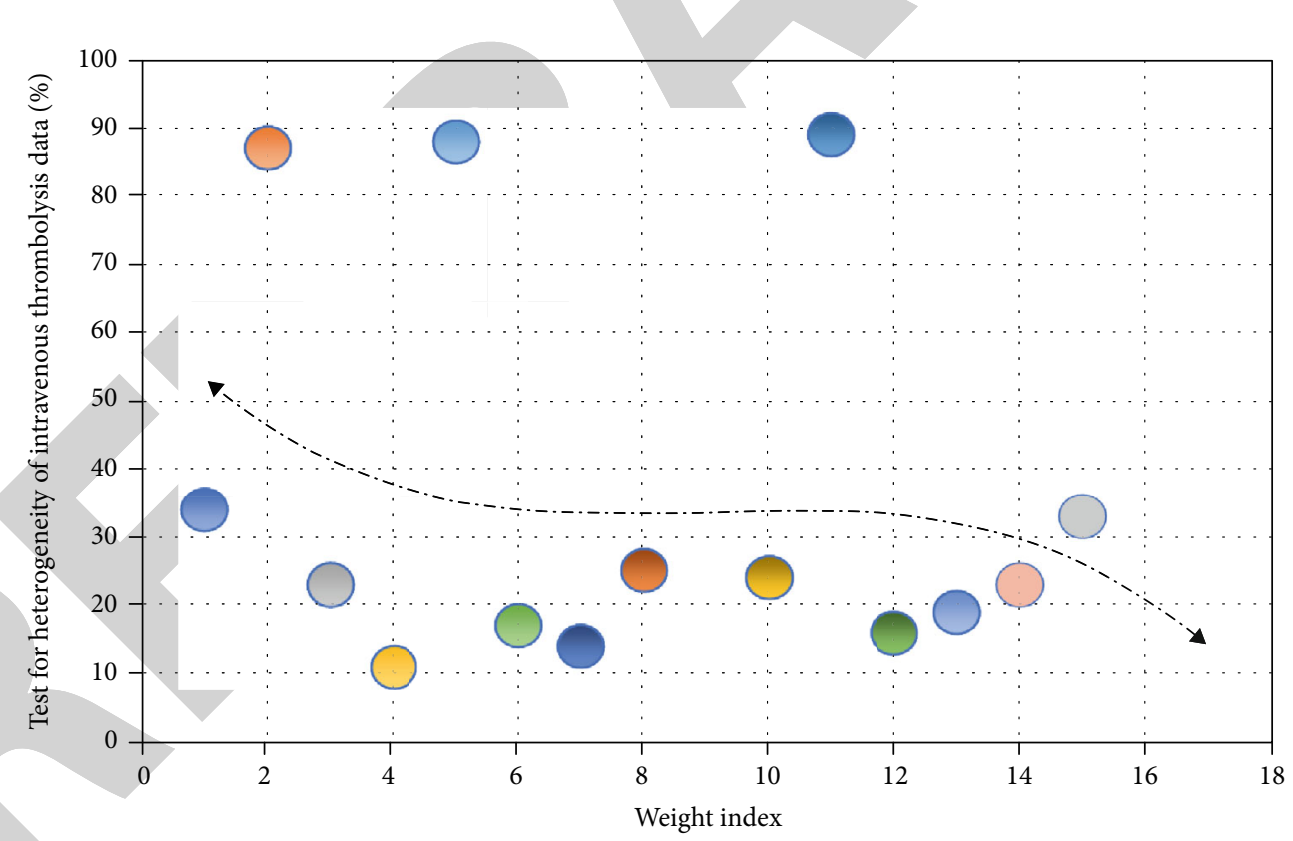

Figure 7: Heterogeneity test of intravenous thrombolysis data.

respectively, $P=0.304$. There was no significant difference in the distribution of high signal between the two groups, and the plaque in the direction of perforating vessels was significantly higher in the drug group than in the surgery group. According to whether the diseased blood vessel is symptomatic or not, T1 hyperintensity accounted for 11/40 (27.5\%) and $0 / 12(0 \%)$ in the symptomatic and nonsymptomatic groups, respectively, $P=0.05$; group accounted for $15 / 40$ $(37.5 \%)$ and $6 / 12(50 \%), P=0.439$; plaque enhancement in the two groups accounted for 24/40 (60\%) and 6/12 (50\%), $P=0.539$. The ratio of $\mathrm{T} 1$ hyperintensity in the symptomatic group was significantly higher than that in the nonsymptomatic group, while the proportion of plaques in the direction of perforating vessels and plaque enhancement in each group was not statistically different between the two groups.

4.3. Case Application and Analysis. Among the outcome indicators of intravenous thrombolysis, the $I^{2}$ values of the 
TABLE 2: Statistical distribution of outcome indicators of intravenous thrombolysis.

\begin{tabular}{lcccc}
\hline Action number & Outcome & Factors & Action number & Outcome \\
\hline 1 & Hold high & 0.11 & 8 & Squat down and raise your hand \\
2 & Hold high & 0.21 & 9 & Squat down and raise your hand \\
3 & Hold high & 0.14 & 10 & Squat down and raise your hand \\
4 & Flat pendulum & 0.15 & 11 & Bend over \\
5 & Flat pendulum & 0.09 & 12 & Bend over \\
6 & Flat pendulum & 0.26 & 13 & Bend over \\
7 & Swing to the effect & 0.31 & 14 & Sit down \\
\hline
\end{tabular}

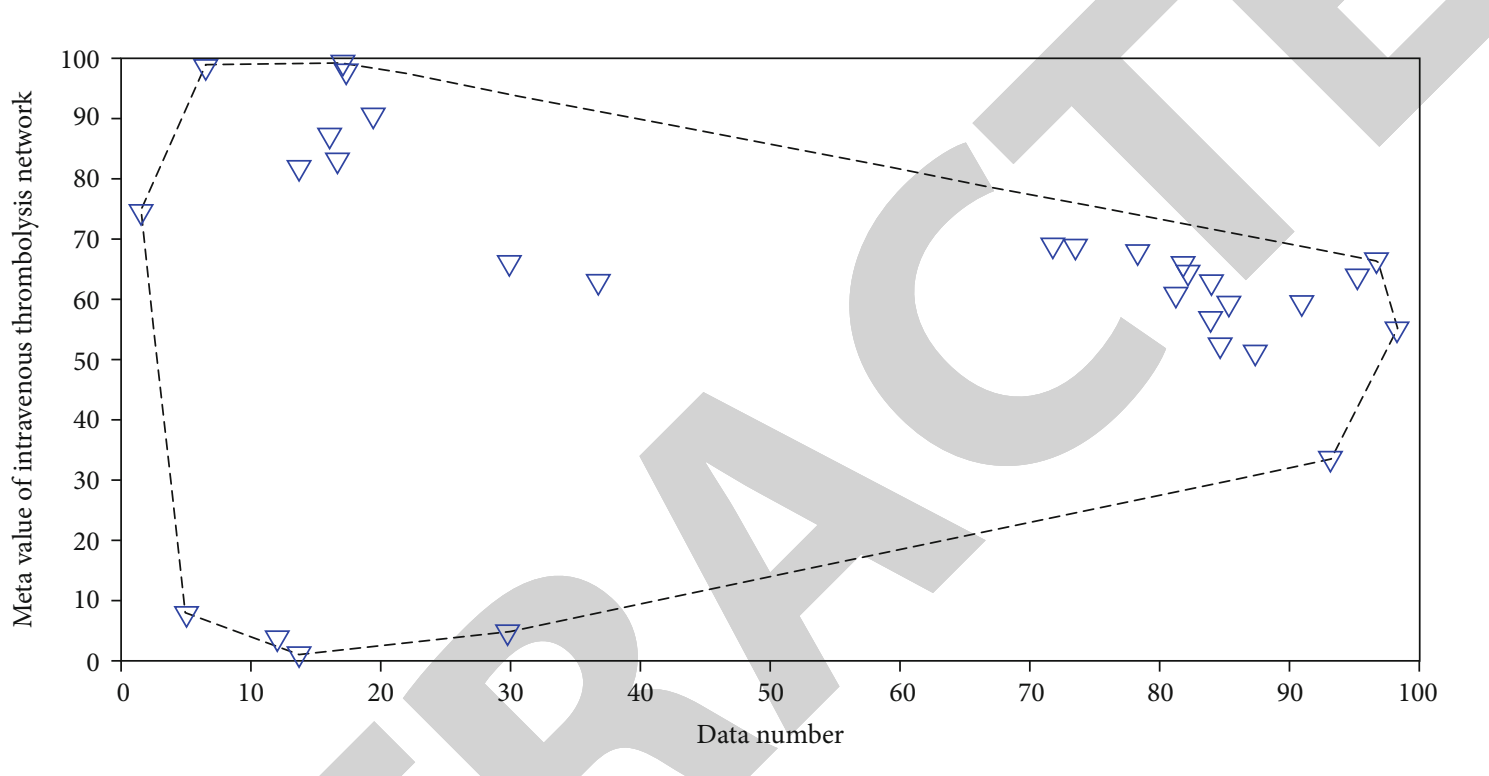

FIGURE 8: Meta-analysis of intravenous thrombolysis network.

surgical success rate and the incidence of in-stent restenosis were $80 \%$ and $52.3 \%$, respectively. Random mode was used, and the $I^{2}$ values of other indicators were less than 50\%, and the fixed mode was used. The single group rate was analyzed by adding weights, and the overall surgical success rate was $96.75 \%$, the 30 -day endpoint event rate was $8.75 \%$, the 1 year endpoint event rate was $13 \%$, and the overall mortality rate was $2.98 \%$. In-stent restenosis occurred. The rate was $21.76 \%$, symptomatic restenosis accounted for $6.5 \%$ of the total number of observations, and symptomatic restenosis accounted for $26.06 \%$ of the number of restenosis. The difference in hypertension $(x=13.210, P<0.05)$ among patients with different modified types was statistically significant. Through pairwise comparisons between groups, it was found that the proportion of AT patients with hyperlipidemia was higher than that of other types of patients, and the difference was statistically significant $\left(P^{\prime}<0.005\right)$. When the results of meta-analysis are heterogeneous, sensitivity analysis is used to deal with them. The method compares different effect models and transforms the random-effects model and the fixed-effects model for meta-analysis. If the before and after results of the sensitivity analysis have not substantially changed, it means that the direct metaanalysis results are more credible. If the sensitivity analysis yields different results, it indicates that there are potentially important factors related to the effect of the intervention. Table 2 shows the statistical distribution of the outcome indicators of intravenous thrombolysis.

The final meta-analysis findings are shown using a forest map. The forest map is constructed using the effect size and confidence interval, and the numerical results are graphed. The vertical line indicates if there is a statistically significant difference in the experimental data. The line depicts the experimental findings' confidence interval. If the combined result is on the left side of the vertical line, the intervention factor has the potential to lower illness incidence. On the contrary, it may enhance the disease's prevalence. When it is vertical, it shows that there is no association between the intervention element and the onset of the illness. That is, the $Z$ value and the $P$ value are used to judge whether the effect size of multiple studies is statistically significant. Generally, the difference of the $P$ value $<0.05$ is considered to be statistically significant. This study is based on the Bayesian algorithm. The convergence is evaluated by running 4 Markov chains. The number of iterations is 50000, the first 20000 annealing; after 50000 iterations, the convergence is good, the PSRF display is close to 1 , and the result is stable. Therefore, the consistency model can be used for mesh meta-analysis. Figure 8 is a meta-analysis of intravenous thrombolysis mesh. 
There was no significant difference between the two groups in the baseline comparison. For the primary endpoint, the incidence within 30 days of the two groups was $4 / 48(8.33 \%)$ and $14 / 172(8.14 \%)$, and the 1-year incidence was $7 / 48(14.58 \%)$ and $28 / 172(16.28 \%)$, the incidence during the total follow-up period was $10 / 48(20.83 \%)$ and $39 /$ $172(22.67 \%)$, and there was no statistical difference. The primary and secondary endpoints were compared by the Kaplan-Meier method and log-rank test. The chi-square values of the two groups were 0.073 and 0.133 , and the $P$ values were 0.787 and 0.716 , respectively. The "Cochrane Risk Bias Assessment Tool” of the Cochrane System Review Manual is used to evaluate the quality of the literature, and the bias risk is evaluated from 6 areas: (1) sequence generation; (2) allocation hiding; (3) evaluation of subjects, researchers, and results; (4) incomplete result data (loss to follow-up bias); (5) whether to report the results of the study selectively; and (6) whether there are other sources of bias. The evaluation of each indicator adopts three levels of "low degree," "unclear," and "high" to determine. There was no significant difference in survival time between the two groups. Kaplan-Meier analysis showed that among the secondary endpoints, the median survival time (95\% confidence interval) of the surgery group and the drug group was $47.082(38.509,55.656)$ and $55.916(50.096,61.736)$, respectively, and there was no statistical difference between the two groups. Multivariate Cox stepwise regression analysis showed that hypertension, hyperlipidemia, history of peripheral arterial disease, acute cerebral infarction, heart disease, hemorrhage, and diabetes were independent risk factors for secondary endpoint events. It can be seen from the cumulative survival function that intravascular interventional therapy for symptomatic intracranial atherosclerotic severe stenosis is better than drug therapy alone.

\section{Conclusion}

For the meta-analysis of the effectiveness and safety of intravenous thrombolysis in patients with acute cerebral infarction, this paper uses standardized mean difference (SMD) and 95\% confidence interval (CI) to evaluate the effect of rTMS. The Stata 14.1 software was used to analyze the stability, sensitivity, and heterogeneity of the research results, and the Cochrane risk of bias tool was used to evaluate the quality of the research. Meta-analysis as it is now used is concerned with comparing the effects of two therapies. In comparison, a networked meta-analysis using the Bayesian technique may include all randomized controlled trials into a single analysis while doing direct and indirect comparisons of various treatment methods. Indirect comparison and rating of treatment plans were performed to determine the most effective course of action. To this purpose, direct and indirect comparative analyses are performed to assess the effectiveness and bleeding risk of different antiplatelet therapies in order to provide a foundation for more informed clinical choices. Except for the CE subtype, the findings of this research indicate that males are more likely to have the other subtypes. This may be due to the higher risk factors for ischemic acute cerebral infarction in men, such as high blood pressure, smoking, coronary artery disease, dyslipidemia, and intravenous thrombolysis. In terms of age, the results of this study show that the age of onset of each subtype is mostly 65 to 70 years old, with fewer young and middle-aged patients, indicating that the incidence of ischemic cerebral acute cerebral infarction increases significantly with age. Regarding the improvement of daily living ability, the subjects in the repetitive transcranial magnetic stimulation combined with the traditional rehabilitation treatment group were better than the single use of the traditional rehabilitation treatment group $(\mathrm{SMD}=0.580$; 95\% CI: $0.377-$ $0.783)$. For the before and after comparison of the severity of acute cerebral infarction, subjects in the repeated transcranial magnetic stimulation therapy combined with traditional rehabilitation therapy group had a more significant decrease in the degree of acute cerebral infarction than the traditional rehabilitation therapy group alone $(\mathrm{SMD}=-0.555 ; 95 \% \mathrm{CI}$ : -0.813 to -0.298$)$.

\section{Data Availability}

The data used to support the findings of this study are included within the article.

\section{Conflicts of Interest}

The authors declare that they have no conflicts of interest.

\section{References}

[1] M. Waqas, C. C. Kuo, R. H. Dossani et al., "Mechanical thrombectomy versus intravenous thrombolysis for distal largevessel occlusion: a systematic review and meta-analysis of observational studies," Neurosurgical Focus, vol. 51, no. 1, p. E5, 2021.

[2] Y. ZHAO and M. TALHA, "Evaluation of food safety problems based on the fuzzy comprehensive analysis method," Food Science and Technology, 2021.

[3] J. Wu, L. Wang, X. Dong et al., "Chinese herbal injections combined with rt-PA intravenous thrombolysis for acute ischemic stroke," Medicine, vol. 100, no. 10, p. e25004, 2021.

[4] B. Huang, F. Qian, X. Fan et al., "Efficacy and safety of intravenous thrombolysis with alteplase for treating acute ischemic stroke at different time windows," Medicine, vol. 99, no. 52, pp. e23620-e23624, 2020.

[5] J. W. Cheng, X. J. Zhang, L. S. Cheng et al., "Low-dose tissue plasminogen activator in acute ischemic stroke: a systematic review and meta-analysis," Journal of Stroke and Cerebrovascular Diseases, vol. 27, no. 2, pp. 381-390, 2018.

[6] J. Liu, X. Hu, Y. Wang, X. Guan, J. Chen, and H. Liu, "The safety and effectiveness of early anti-platelet therapy after alteplase for acute ischemic stroke: a meta-analysis," Journal of Clinical Neuroscience, vol. 91, pp. 176-182, 2021.

[7] O. M. Dumitrascu, J. F. Shen, M. Kurli et al., "Is intravenous thrombolysis safe and effective in central retinal artery occlusion? A critically appraised topic," The Neurologist, vol. 22, no. 4, pp. 153-156, 2017.

[8] L. Huang, Y. Wang, and R. Zhang, "Intravenous thrombolysis in patients with central retinal artery occlusion: a systematic review and meta-analysis," Journal of Neurology, vol. 10, pp. 6-9, 2021. 
[9] X. Li, H. du, Z. Song et al., "Efficacy and safety of sonothrombolysis in patients with acute ischemic stroke: a systematic review and meta-analysis," Journal of the Neurological Sciences, vol. 416, article 116998, 2020.

[10] S. Vidale, M. Romoli, D. Consoli, and E. C. Agostoni, "Bridging versus direct mechanical thrombectomy in acute ischemic stroke: a subgroup pooled meta-analysis for time of intervention, eligibility, and study design," Cerebrovascular Diseases, vol. 49, no. 2, pp. 223-232, 2020.

[11] D. Flynn, R. Francis, K. Halvorsrud et al., "Intra-arterial mechanical thrombectomy stent retrievers and aspiration devices in the treatment of acute ischaemic stroke: a systematic review and meta-analysis with trial sequential analysis," European Stroke Journal, vol. 2, no. 4, pp. 308-318, 2017.

[12] J. W. Wong, L. Churilov, R. Dowling et al., "Safety of endovascular thrombectomy for acute ischaemic stroke in anticoagulated patients ineligible for intravenous thrombolysis," Cerebrovascular Diseases, vol. 46, no. 5-6, pp. 193-199, 2019.

[13] J. Lin, Y. Liang, and J. Lin, "Endovascular therapy versus intravenous thrombolysis in cervical artery dissection-related ischemic stroke: a meta-analysis," Journal of Neurology, vol. 267, no. 6, pp. 1585-1593, 2020.

[14] K. Phan, A. A. Dmytriw, J. Maingard et al., "Endovascular thrombectomy alone versus combined with intravenous thrombolysis," World Neurosurgery, vol. 108, pp. 850-858.e2, 2017.

[15] R. Hu, Y. Guo, Y. Lin et al., "Safety and efficacy of edaravone combined with alteplase for patients with acute ischemic stroke: a systematic review and meta-analysis," Die Pharmazie-An International Journal of Pharmaceutical Sciences, vol. 76, no. 2-3, pp. 109-113, 2021.

[16] K. Wang, D. Zhang, J. Wu, S. Liu, X. Zhang, and B. Zhang, "A comparative study of Danhong injection and Salvia miltiorrhiza injection in the treatment of cerebral infarction," Medicine, vol. 96, no. 22, p. e7079, 2017.

[17] A. H. Katsanos, A. Safouris, A. Sarraj et al., "Intravenous thrombolysis with tenecteplase in patients with large vessel occlusions," Stroke, vol. 52, no. 1, pp. 308-312, 2021.

[18] G. Li, R. Huang, W. Li, X. Zhang, and G. Bi, "Mechanical thrombectomy with second-generation devices for acute cerebral middle artery M2 segment occlusion: a meta-analysis," Interventional Neuroradiology, vol. 26, no. 2, pp. 187-194, 2020.

[19] B. Karaszewski, A. Wyszomirski, B. Jabłoński, D. J. Werring, and D. Tomaka, "Efficacy and safety of intravenous rtPA in ischemic strokes due to small-vessel occlusion: systematic review and meta-analysis," Translational Stroke Research, vol. 12, no. 3, pp. 406-415, 2021.

[20] J. Wang, X. Fang, D. Wang, and Y. Xiao, "Effect of intravenous thrombolysis with alteplase on clinical efficacy, inflammatory factors, and neurological function in patients with acute cerebral infarction," Brazilian Journal of Medical and Biological Research, vol. 54, no. 5, p. e10000, 2021.

[21] G. Kumar and A. V. Alexandrov, "Vasospasm surveillance with transcranial Doppler sonography in subarachnoid hemorrhage," Journal of Ultrasound in Medicine, vol. 34, no. 8, pp. 1345-1350, 2015. 